\title{
Relationships of dietary patterns with body composition in older adults differ by gender and PPAR- $\gamma$ Pro12Ala genotype
}

\author{
Amy L. Anderson • Tamara B. Harris • Denise K. Houston • \\ Frances A. Tylavsky $\cdot$ Jung Sun Lee $\cdot$ Deborah E. Sellmeyer • \\ Nadine R. Sahyoun
}

Received: 27 October 2009/ Accepted: 28 January 2010/Published online: 21 February 2010

(c) The Author(s) 2010. This article is published with open access at Springerlink.com

\begin{abstract}
Purpose Dietary patterns may better capture the multifaceted effects of diet on body composition than individual nutrients or foods. The objective of this study was to investigate the dietary patterns of a cohort of older adults, and examine relationships of dietary patterns with body composition. The influence of a polymorphism in the peroxisome proliferator-activated receptor- $\gamma$ (PPAR- $\gamma$ ) gene was considered.

Methods The Health, Aging and Body Composition (Health ABC) Study is a prospective cohort study of 3,075
\end{abstract}

For the Health ABC Study.

\section{A. L. Anderson $(\bowtie) \cdot N$. R. Sahyoun}

Department of Nutrition and Food Science,

University of Maryland, 0112 Skinner Building,

College Park, MD 20742, USA

e-mail: amylouiseanderson@yahoo.com

T. B. Harris

National Institute on Aging, NIH,

Bethesda, MD 20892, USA

\section{K. Houston}

Sticht Center on Aging, Wake Forest University,

Winston-Salem, NC 27157, USA

F. A. Tylavsky

Department of Preventive Medicine,

University of Tennessee, Memphis, TN 38103, USA

J. S. Lee

Department of Foods and Nutrition,

University of Georgia, Athens, GA 30602, USA

D. E. Sellmeyer

Division of Endocrinology, University of California,

San Francisco, CA 94115, USA older adults. Participants' body composition and genetic variation were measured in detail. Food intake was assessed with a semi-quantitative food frequency questionnaire (Block Dietary Data Systems, Berkeley, CA), and dietary patterns of 1,809 participants with complete data were derived by cluster analysis.

Results Six clusters were identified, including a 'Healthy foods' cluster characterized by higher intake of low-fat dairy products, fruit, whole grains, poultry, fish and vegetables. An interaction was found between dietary patterns and PPAR- $\gamma$ Pro12Ala genotype in relation to body composition. While Pro/Pro homozygous men and women in the 'Healthy foods' cluster did not differ significantly in body composition from those in other clusters, men with the Ala allele in the 'Healthy foods' cluster had significantly lower levels of adiposity than those in other clusters. Women with the Ala allele in the 'Healthy foods' cluster differed only in right thigh intermuscular fat from those in other clusters.

Conclusions Relationships between diet and body composition in older adults may differ by gender and by genetic factors such as PPAR- $\gamma$ Pro12Ala genotype.

Keywords Diet - Dietary patterns - Body composition . Older adults $\cdot$ PPAR $-\gamma$ Pro12Ala genotype

\section{Introduction}

While obesity is considered a major health risk, the regional distribution of body fat may be of greater consequence than overall body fat. Excess fat in the abdominal visceral area in particular has been associated with higher risk for multiple metabolic complications and chronic diseases, as well as increased mortality [1-7]. 
Dietary pattern analysis examines the overall diet, and thus takes into account correlation among nutrient intakes as well as nutrient-nutrient interactions. Compared to a focus on individual nutrients or foods, dietary pattern analysis may better capture the complexity of dietary exposure thought to affect body composition.

Both environmental and genetic factors likely influence body composition and body fat distribution [8, 9]. The peroxisome proliferator-activated receptor- $\gamma$ (PPAR- $\gamma$ ) is expressed in adipose tissue and regulates adipocyte differentiation and gene expression in adipocytes. A common polymorphism (Pro12Ala) in the PPAR- $\gamma 2$ isoform of the PPAR- $\gamma$ gene has been linked to greater adiposity in some studies [10-14], but not in others [15-18]. Polymorphisms in genes such as the PPAR- $\gamma$ gene may need to be considered when examining the influence of diet on body composition.

The purpose of the current study was to determine the main dietary patterns of a cohort of older adults, and to examine whether dietary pattern groups differed in measures of body composition, including abdominal visceral fat. A secondary goal was to investigate the possible influence of variation in the PPAR- $\gamma$ gene on the relationship between diet and body composition.

\section{Subjects and methods}

\section{Study population}

Participants age 70-79 were recruited for the Health, Aging and Body Composition (Health ABC) Study, a prospective cohort study, from a random sample of white Medicareeligible residents and from all age-eligible black residents of selected areas of Pittsburgh, Pennsylvania, and Memphis, Tennessee. Individuals were eligible for Health ABC if they planned to remain in the area for at least 3 years and reported no life-threatening cancers and no difficulty with basic activities of daily living, walking $1 / 4$ mile or climbing ten steps. Those who used assistive devices were excluded, as were participants in any research studies which involved a lifestyle intervention. Protocols were approved by institutional review boards at both study sites, and participants provided written, informed consent. An interview on behavior, health status, and social, demographic and economic factors, and a clinical examination of body composition, biochemical variables, weight-related health conditions and physical function were administered between 1997 and 1998, with annual follow-up assessments.

Data from baseline and year 2 of the Health ABC study were used in the current analyses. The sample size for most analyses in this study was 1,809, after excluding participants who did not have a dietary assessment ( $n=343$ ); those diagnosed with type 2 diabetes before dietary intake was assessed $(n=662)$; men who reported an energy intake of $<800 \mathrm{kcal} /$ day or $>4,000 \mathrm{kcal} /$ day and women who reported an energy intake of $<500 \mathrm{kcal} /$ day or $>3,500 \mathrm{kcal} /$ day $(n=77)$; and those with incomplete information on other relevant measures $(n=184)$. Further exclusions were made in some analyses if outcome variables of interest were missing or implausible.

\section{Dietary assessment}

Food intake was measured in year 2 of the Health ABC study with a 108-item semi-quantitative food frequency questionnaire (FFQ). This FFQ was designed specifically for the Health ABC study by Block Dietary Data Systems (Berkeley, CA), based on reported intakes of non-Hispanic white and black residents of the Northeast and South over age 65 in the third National Health and Nutrition Examination Survey. The FFQ was administered by a trained dietary interviewer, and interviews were periodically monitored to assure quality and consistency. Wood blocks, real food models, and flash cards were used to help participants estimate portion sizes. Nutrient and food group intakes were determined by Block Dietary Data Systems, as were participants' dietary GI and GL values, as described previously [19]. A healthy eating index (HEI) score, which reflects how well the diet conforms to the recommendations of the Dietary Guidelines for Americans and the Food Guide Pyramid, was also calculated for each participant [20].

In this study, individuals were grouped according to their overall dietary patterns by cluster analysis based on methods used in previous studies [21, 22]. The purpose of the cluster analysis was to place individuals into mutually exclusive groups such that persons in a given cluster had similar diets which differed from those of persons in other clusters. First, the 108 FFQ food items were consolidated into 40 food groups according to similarity in nutrient content. The percentage of energy contributed by each food group for each participant was calculated and used in the cluster analysis. The reason for this standardization was to account for differences in total energy needs due to gender, age, body size and level of physical activity.

The FASTCLUS procedure in SAS (version 9.1; SAS Institute Inc., Cary, NC) was used to generate dietary pattern clusters. This procedure requires the number of clusters to be specified in advance, and generates mutually exclusive clusters by comparing Euclidean distances between each subject and each cluster center in an interactive process using a K-means method. To determine the most appropriate number of clusters, two to eight cluster 
solutions were run. Plots of $R^{2}$ by the number of clusters and of the ratio of between-cluster variance to withincluster variance by the number of clusters were examined. A set of six clusters was selected, as this solution most clearly identified distinct and nutritionally meaningful dietary patterns while maintaining a reasonable sample size in each group for subsequent regression analyses. Mean percent energy contributions from food groups were examined according to dietary pattern clusters. Clusters were named according to food groups that on average contributed relatively more to total energy intake.

\section{Measures of body composition}

At baseline of the Health ABC study, participants underwent axial computed tomography scanning of the abdomen and thigh. Abdominal visceral and subcutaneous fat and thigh intermuscular fat were quantified from scans performed on a General Electric 9800 Advantage in Pittsburgh and a Siemens Somatron and Picker PQ2000S in Memphis. Data from computed tomography scans were analyzed at the University of Colorado Health Sciences Center according to a standardized protocol [23]. Total fat mass and lean mass were assessed at baseline and year 2 by dual energy X-ray absorptiometry (Hologic QDR 4500A, software version 8.21, Hologic, Waltham, MA). Abdominal sagittal diameter was measured at baseline with a Holtain-Kahn abdominal calliper (Holtain Ltd, UK), and abdominal circumference was measured at baseline with a tape measure at the level of the largest circumference. Weight in kilograms was measured annually with a standard balance beam scale, and height in meters measured twice at baseline with a Harpenden stadiometer (Holtain Ltd, Crosswell, UK). After averaging the two height measurements, BMI $\left(\mathrm{kg} / \mathrm{m}^{2}\right)$ was calculated as weight divided by the square of height.

\section{Sociodemographic and lifestyle variables}

Sociodemographic variables including age, gender, selfidentified racial group and education, and lifestyle variables including smoking status, alcohol consumption, and physical activity were assessed at baseline of the Health ABC study. Lifetime pack-years of cigarette smoking were calculated by multiplying cigarette packs smoked per day by the number of years of smoking. Physical activity was evaluated by a standardized questionnaire specifically designed for the Health $\mathrm{ABC}$ study. This questionnaire was derived from the leisure time physical activity questionnaire and included activities commonly performed by older adults [24]. The frequency, duration, and intensity of specific activities were determined, and approximate metabolic equivalent unit (MET) values assigned to each activity category to estimate weekly energy expenditure.
Genotyping

The Health $\mathrm{ABC}$ cohort was genotyped, using polymerase chain reaction restriction fragment length polymorphism analysis (PCR-RFLP), for the Pro12Ala polymorphism of the PPAR- $\gamma$ gene by Beamer et al. [25]. In the current study population, PPAR- $\gamma$ Pro12Ala genotype frequencies were found to be in Hardy-Weinberg equilibrium.

\section{Statistical analysis}

Characteristics of men and women were examined by dietary pattern cluster, and each cluster was compared to the 'Healthy foods' cluster with Dunnett's test for continuous variables and chi-square test for categorical variables. Multiple regression models were constructed to compare mean body composition measures of each cluster to the 'Healthy foods' cluster, controlled for possible confounding factors including age, race, clinical site, education, physical activity, smoking and total calorie intake. The interaction of dietary pattern and gender was tested, as was the interaction of dietary pattern and PPAR $-\gamma$ Pro12Ala genotype. As these interactions were found to be statistically significant, subsequent analyses were conducted by gender and additionally by PPAR- $\gamma$ Pro12Ala genotype. Statistical significance was set at $P \leq 0.05$, and analyses were performed using SAS (version 9.1; SAS Institute Inc., Cary, NC).

\section{Results}

Six clusters were identified: (1) 'Meat, snacks, fats and alcohol' $(n=480)$; (2) 'Sweets and desserts' $(n=257)$; (3) 'Refined grains' $(n=247)$; (4) 'Breakfast cereal' $(n=273)$; (5) 'Healthy foods' $(n=306)$; and (6) 'Highfat dairy products' $(n=246)$. Table 1 shows mean percent energy contributions from food groups to dietary pattern clusters. The 'Healthy foods' cluster was characterized by relatively higher intake of low-fat dairy products, fruit, whole grains, poultry, fish and vegetables, and lower consumption of red meat, sweets, added fats and high-calorie drinks.

Tables 2 and 3 show characteristics of men and women by dietary pattern cluster. The 'Healthy foods' cluster had a significantly higher percent of women than any of the other five clusters. Both men and women in the 'Healthy foods' cluster had a higher percent energy intake from protein, lower percent energy from total fat and saturated fat, and higher intake of fiber than those in other clusters. The 'Healthy foods' cluster also had a higher percent energy from carbohydrate, and a lower dietary glycemic index and glycemic load than most other clusters. In addition, the 
Table 1 Percent energy contribution from selected food groups to total energy intake across the six dietary pattern clusters

\begin{tabular}{|c|c|c|c|c|c|c|}
\hline \multirow[t]{2}{*}{ Food group } & \multicolumn{6}{|c|}{ Percent energy contribution ${ }^{a}$} \\
\hline & $\begin{array}{l}\text { Healthy foods } \\
(n=306)\end{array}$ & $\begin{array}{l}\text { Meat, snacks, } \\
\text { fats and alcohol } \\
(n=480)\end{array}$ & $\begin{array}{l}\text { Sweets and } \\
\text { desserts } \\
(n=257)\end{array}$ & $\begin{array}{l}\text { Refined } \\
\text { grains } \\
(n=247)\end{array}$ & $\begin{array}{l}\text { Breakfast } \\
\text { cereal } \\
(n=273)\end{array}$ & $\begin{array}{l}\text { High-fat dairy } \\
\text { products } \\
(n=246)\end{array}$ \\
\hline Processed meat & $1.7 \pm 2.0$ & $4.0 \pm 3.3$ & $2.6 \pm 2.5$ & $3.6 \pm 3.2$ & $2.4 \pm 2.3$ & $3.0 \pm 3.0$ \\
\hline Meat & $2.8 \pm 2.7$ & $4.0 \pm 3.1$ & $3.4 \pm 2.7$ & $3.5 \pm 2.9$ & $3.5 \pm 3.1$ & $3.7 \pm 3.4$ \\
\hline Fish and other seafood & $2.7 \pm 2.7$ & $1.7 \pm 2.1$ & $1.3 \pm 1.6$ & $1.4 \pm 2.1$ & $2.0 \pm 2.5$ & $1.3 \pm 1.5$ \\
\hline Poultry (not fried) & $3.4 \pm 4.3$ & $2.2 \pm 2.7$ & $2.0 \pm 2.3$ & $2.0 \pm 2.5$ & $2.0 \pm 2.0$ & $1.9 \pm 2.4$ \\
\hline Fried poultry & $0.4 \pm 1.0$ & $1.5 \pm 2.8$ & $0.6 \pm 1.1$ & $1.1 \pm 1.9$ & $0.6 \pm 1.1$ & $0.9 \pm 1.8$ \\
\hline Low-fat dairy products & $9.4 \pm 6.7$ & $1.0 \pm 2.0$ & $1.8 \pm 3.0$ & $1.6 \pm 3.2$ & $2.7 \pm 3.9$ & $0.5 \pm 1.4$ \\
\hline Higher-fat dairy products & $3.5 \pm 2.8$ & $5.1 \pm 2.9$ & $6.2 \pm 4.5$ & $5.5 \pm 3.9$ & $6.3 \pm 3.8$ & $16.7 \pm 5.6$ \\
\hline Beer & $0.3 \pm 1.4$ & $1.4 \pm 4.5$ & $0.3 \pm 1.3$ & $0.4 \pm 2.3$ & $0.5 \pm 1.9$ & $0.4 \pm 1.9$ \\
\hline Liquor & $0.6 \pm 2.4$ & $1.1 \pm 3.6$ & $0.6 \pm 2.1$ & $0.4 \pm 1.6$ & $0.7 \pm 2.0$ & $0.6 \pm 1.9$ \\
\hline Fruit & $8.2 \pm 5.0$ & $4.0 \pm 3.1$ & $3.6 \pm 3.0$ & $3.9 \pm 3.3$ & $4.7 \pm 3.8$ & $4.3 \pm 3.7$ \\
\hline Dark green vegetables & $0.4 \pm 0.5$ & $0.2 \pm 0.3$ & $0.2 \pm 0.2$ & $0.3 \pm 0.3$ & $0.2 \pm 0.3$ & $0.3 \pm 0.3$ \\
\hline Dark yellow vegetables & $1.1 \pm 1.4$ & $0.7 \pm 1.0$ & $0.7 \pm 1.1$ & $0.9 \pm 1.4$ & $0.7 \pm 0.7$ & $0.8 \pm 1.0$ \\
\hline Other vegetables & $1.4 \pm 1.4$ & $1.1 \pm 1.3$ & $1.1 \pm 1.2$ & $1.3 \pm 1.2$ & $1.2 \pm 1.1$ & $1.3 \pm 1.4$ \\
\hline Whole grains & $5.8 \pm 5.4$ & $3.2 \pm 3.5$ & $2.4 \pm 2.8$ & $2.1 \pm 3.5$ & $2.7 \pm 3.0$ & $3.1 \pm 3.8$ \\
\hline Cold breakfast cereal: fiber/bran & $2.9 \pm 3.5$ & $1.5 \pm 2.4$ & $1.5 \pm 2.6$ & $1.0 \pm 1.9$ & $3.7 \pm 4.9$ & $2.0 \pm 2.9$ \\
\hline Other cold breakfast cereal & $6.7 \pm 4.3$ & $4.5 \pm 3.4$ & $5.3 \pm 4.2$ & $4.1 \pm 4.3$ & $18.4 \pm 6.3$ & $5.9 \pm 4.2$ \\
\hline Refined grains & $9.5 \pm 5.0$ & $10.7 \pm 4.3$ & $10.1 \pm 5.4$ & $25.3 \pm 6.8$ & $8.7 \pm 4.7$ & $11.0 \pm 4.9$ \\
\hline Rice, pasta and mixed dishes & $4.2 \pm 4.2$ & $4.0 \pm 3.8$ & $3.0 \pm 2.7$ & $2.9 \pm 2.7$ & $3.0 \pm 2.5$ & $2.9 \pm 2.6$ \\
\hline Snacks & $1.4 \pm 2.9$ & $2.8 \pm 5.1$ & $2.1 \pm 3.9$ & $1.5 \pm 2.6$ & $1.4 \pm 2.5$ & $1.7 \pm 3.1$ \\
\hline Nuts & $3.3 \pm 4.0$ & $4.7 \pm 6.7$ & $3.0 \pm 3.6$ & $3.2 \pm 3.9$ & $2.6 \pm 3.9$ & $3.2 \pm 4.0$ \\
\hline High-calorie drinks & $0.8 \pm 1.8$ & $4.0 \pm 5.2$ & $1.7 \pm 3.0$ & $2.7 \pm 4.2$ & $2.1 \pm 3.5$ & $2.9 \pm 4.9$ \\
\hline Mayonnaise and salad dressing & $3.0 \pm 2.8$ & $4.9 \pm 4.2$ & $3.0 \pm 2.7$ & $2.9 \pm 2.7$ & $3.6 \pm 3.2$ & $3.9 \pm 3.2$ \\
\hline Sweets and desserts & $6.3 \pm 4.7$ & $7.8 \pm 4.7$ & $26.2 \pm 8.8$ & $8.0 \pm 5.5$ & $7.2 \pm 5.0$ & $6.7 \pm 4.7$ \\
\hline Miscellaneous fats & $3.6 \pm 3.5$ & $5.9 \pm 4.5$ & $4.0 \pm 3.5$ & $5.3 \pm 4.1$ & $3.8 \pm 3.2$ & $4.6 \pm 3.7$ \\
\hline
\end{tabular}

Mean $\pm \mathrm{SD}$, unless otherwise specified

${ }^{a}$ The highest and lowest percent energy contributions from each food group across the six dietary pattern clusters are in bold

'Healthy foods' cluster had a significantly higher HEI score than any other cluster.

Table 4 shows selected body composition measures of men according to dietary pattern cluster. After adjustment for age, race, clinical site, education, physical activity, smoking and total calorie intake, men in the 'Healthy foods' cluster had a significantly lower total percent body fat than those in the 'Meat, snacks, fats and alcohol' and 'Breakfast cereal' clusters (Table 4). Men in the 'Healthy foods' cluster also had less abdominal visceral fat than those in the 'Breakfast cereal' cluster. No differences were found between men in the 'Healthy foods' and other clusters in BMI, abdominal circumference, sagittal diameter, abdominal subcutaneous fat, right thigh intermuscular fat or total lean body mass. Women in the 'Healthy foods' cluster showed no significant differences in any measures of body composition from any other clusters (results not shown).

Tables 5 and 6 show selected body composition measures of men and women by PPAR- $\gamma$ genotype according to dietary pattern cluster. Pro/Pro homozygous men and women in the 'Healthy foods' cluster did not differ significantly in any measures of body composition from those in other clusters, after adjustment for age, race, clinical site, education, physical activity, smoking and total calorie intake (Tables 5, 6). Conversely, men with the Ala allele in the 'Healthy foods' cluster differed significantly in almost all measures of body composition from those in other clusters (Table 5). Men with the Ala allele in the 'Healthy foods' cluster had a significantly lower BMI, total percent body fat, sagittal diameter, and abdominal visceral and subcutaneous fat areas than those in the 'Meat, snacks, fats and alcohol' and 'Breakfast cereal' clusters. Men with the Ala allele in the 'Healthy foods' cluster also had a lower total percent body fat and sagittal diameter than those in the 'High-fat dairy products' cluster, and a smaller abdominal circumference than those in the 'Refined grains' cluster. Additionally, men with the Ala allele in the 'Healthy foods' cluster had significantly less right thigh 
Table 2 Characteristics of men by dietary pattern cluster

\begin{tabular}{|c|c|c|c|c|c|c|}
\hline & $\begin{array}{l}\text { Healthy foods } \\
\text { (reference) }\end{array}$ & $\begin{array}{l}\text { Meat, snacks, fats } \\
\text { and alcohol }\end{array}$ & $\begin{array}{l}\text { Sweets and } \\
\text { desserts }\end{array}$ & $\begin{array}{l}\text { Refined } \\
\text { grains }\end{array}$ & $\begin{array}{l}\text { Breakfast } \\
\text { cereal }\end{array}$ & $\begin{array}{l}\text { High-fat dairy } \\
\text { products }\end{array}$ \\
\hline$n$ (\% men in cluster) & $102(33.3)$ & $234\left(48.8^{\mathrm{a}}\right)$ & $123\left(47.9^{\mathrm{a}}\right)$ & $122\left(49.4^{\mathrm{a}}\right)$ & $145\left(53.1^{\mathrm{a}}\right)$ & $105\left(42.7^{\mathrm{a}}\right)$ \\
\hline \multicolumn{7}{|l|}{ Characteristics } \\
\hline Age $(\text { years })^{\mathrm{b}}$ & $75.3 \pm 0.3$ & $75.0 \pm 0.2$ & $75.7 \pm 0.3$ & $75.1 \pm 0.3$ & $75.3 \pm 0.2$ & $75.5 \pm 0.3$ \\
\hline Race (\% white) & 88.2 & $60.7^{\mathrm{a}}$ & $75.6^{\mathrm{a}}$ & $57.4^{\mathrm{a}}$ & 85.5 & $72.4^{\mathrm{a}}$ \\
\hline Education $\left(\%\right.$ completed high school) ${ }^{\mathrm{c}}$ & 87.3 & $76.1^{\mathrm{a}}$ & 80.5 & $60.7^{\mathrm{a}}$ & 89.7 & 83.8 \\
\hline Smoking (lifetime pack-years) ${ }^{\mathrm{c}}$ & $16.5 \pm 2.3$ & $25.1 \pm 2.1$ & $28.5 \pm 3.2^{\mathrm{a}}$ & $23.9 \pm 2.6$ & $27.7 \pm 2.6^{\mathrm{a}}$ & $27.0 \pm 2.9$ \\
\hline Alcohol (\% any consumption $)^{\mathrm{c}}$ & 68.6 & 69.7 & $49.6^{\mathrm{a}}$ & $46.7^{\mathrm{a}}$ & 68.3 & 66.7 \\
\hline Physical activity (kcal/week) ${ }^{\mathrm{c}}$ & $2,129 \pm 240$ & $1,420 \pm 171^{\mathrm{a}}$ & $1,337 \pm 175^{\mathrm{a}}$ & $1,321 \pm 191^{\mathrm{a}}$ & $1,473 \pm 116$ & $1,255 \pm 156^{\mathrm{a}}$ \\
\hline \multicolumn{7}{|l|}{ PPAR- $\gamma$ Pro12Ala genotype $[n(\%)]^{\mathrm{d}}$} \\
\hline Pro/Pro & $79(79.0)$ & $203\left(87.5^{\mathrm{a}}\right)$ & $95(81.9)$ & $106(88.3)$ & $110(76.9)$ & $72(71.3)$ \\
\hline Ala/Pro and Ala/Ala & $21(21.0)$ & $29\left(12.5^{\mathrm{a}}\right)$ & $21(18.1)$ & $14(11.7)$ & $33(23.1)$ & $29(28.7)$ \\
\hline \multicolumn{7}{|l|}{ Dietary factors ${ }^{\mathrm{b}}$} \\
\hline Total calorie intake (kcal) & $1,848 \pm 53$ & $2,007 \pm 42$ & $2,232 \pm 67^{\mathrm{a}}$ & $1,996 \pm 58$ & $1,885 \pm 48$ & $2,130 \pm 68^{\mathrm{a}}$ \\
\hline$\%$ kcal from carbohydrate & $57.2 \pm 0.7$ & $48.9 \pm 0.5^{\mathrm{a}}$ & $53.4 \pm 0.6^{\mathrm{a}}$ & $53.2 \pm 0.6^{\mathrm{a}}$ & $58.2 \pm 0.6$ & $50.8 \pm 0.7^{\mathrm{a}}$ \\
\hline$\%$ kcal from protein & $16.5 \pm 0.3$ & $14.0 \pm 0.2^{\mathrm{a}}$ & $12.5 \pm 0.2^{\mathrm{a}}$ & $14.1 \pm 0.2^{\mathrm{a}}$ & $14.1 \pm 0.2^{\mathrm{a}}$ & $14.5 \pm 0.2^{\mathrm{a}}$ \\
\hline$\% \mathrm{kcal}$ from fat & $27.0 \pm 0.6$ & $36.0 \pm 0.4^{\mathrm{a}}$ & $35.4 \pm 0.6^{\mathrm{a}}$ & $33.5 \pm 0.6^{\mathrm{a}}$ & $28.1 \pm 0.5$ & $35.3 \pm 0.6^{\mathrm{a}}$ \\
\hline$\%$ kcal from saturated fat & $7.4 \pm 0.2$ & $10.1 \pm 0.1^{\mathrm{a}}$ & $10.4 \pm 0.2^{\mathrm{a}}$ & $9.4 \pm 0.2^{\mathrm{a}}$ & $8.2 \pm 0.2^{\mathrm{a}}$ & $11.8 \pm 0.2^{\mathrm{a}}$ \\
\hline Total dietary fiber $(\mathrm{g})$ & $22.2 \pm 0.8$ & $17.3 \pm 0.5^{\mathrm{a}}$ & $19.1 \pm 0.7^{\mathrm{a}}$ & $17.3 \pm 0.7^{\mathrm{a}}$ & $18.2 \pm 0.6^{\mathrm{a}}$ & $17.3 \pm 0.8^{\mathrm{a}}$ \\
\hline Dietary glycemic index (glucose scale) & $54.5 \pm 0.4$ & $55.2 \pm 0.3$ & $56.3 \pm 0.3^{\mathrm{a}}$ & $59.8 \pm 0.3^{\mathrm{a}}$ & $59.2 \pm 0.2^{\mathrm{a}}$ & $55.5 \pm 0.4$ \\
\hline Dietary glycemic load (glucose scale) & $132.2 \pm 4.5$ & $125.7 \pm 3.0$ & $155.2 \pm 4.7^{\mathrm{a}}$ & $149.0 \pm 4.8^{\mathrm{a}}$ & $151.7 \pm 4.4^{\mathrm{a}}$ & $139.4 \pm 4.6$ \\
\hline Healthy eating index score & $80.9 \pm 0.8$ & $66.3 \pm 0.8^{\mathrm{a}}$ & $64.3 \pm 1.1^{\mathrm{a}}$ & $67.1 \pm 1.1^{\mathrm{a}}$ & $73.3 \pm 0.8^{\mathrm{a}}$ & $66.1 \pm 1.2^{\mathrm{a}}$ \\
\hline
\end{tabular}

Mean \pm SEM, unless otherwise specified

a Significantly different from the 'Healthy foods' cluster, $P \leq 0.05$ (Dunnett's test for continuous variables and chi-square test for categorical variables)

${ }^{b}$ Values from year 2 of the Health ABC study

c Values from baseline of the Health $\mathrm{ABC}$ study

d Genotype information not available for 19 men

intermuscular fat than those in the 'Meat, snacks, fats and alcohol' cluster. On the other hand, women with the Ala allele in the 'Healthy foods' cluster had significantly less right thigh intermuscular fat than those in the 'High-fat dairy products' cluster, but showed no significant differences in any other measures of body composition from any other clusters (Table 6).

\section{Discussion}

In this study of older adults, a variety of distinct dietary patterns were identified. Men in the 'Healthy foods' cluster had a lower total percent body fat than those in the 'Meat, snacks, fats and alcohol' and 'Breakfast cereal' clusters, and less abdominal visceral fat than those in the 'Breakfast cereal' cluster. On the other hand, women in the 'Healthy foods' cluster showed no significant differences in any measures of body composition from any other clusters.
Several other studies have examined dietary patterns of older adults and their associations with adiposity. Ledikwe et al. [26] studied dietary patterns of rural men and women age 66-87 in relation to weight, and showed that those in a low-nutrient-dense cluster, with high intake of breads, sweet breads and desserts, processed meats, eggs, and fats/ oils, were twice as likely to be obese as those in a highnutrient-dense cluster, with high intake of cereals, dark green/yellow vegetables, other vegetables, citrus/melons/ berries, fruit juices, other fruits, milks, poultry, fish, and beans. In the Baltimore Longitudinal Study of Aging, Newby et al. [27] found a dietary pattern high in reducedfat dairy products, fruit, and fiber to be inversely associated with annual change in BMI in women, and inversely associated with annual change in waist circumference in both sexes.

In the current study, dietary patterns were found to interact with PPAR- $\gamma$ Pro12Ala genotype in relation to body composition. Specifically, while Pro/Pro homozygous men and women in the 'Healthy foods' cluster did 
Table 3 Characteristics of women by dietary pattern cluster

\begin{tabular}{|c|c|c|c|c|c|c|}
\hline & $\begin{array}{l}\text { Healthy foods } \\
\text { (reference) }\end{array}$ & $\begin{array}{l}\text { Meat, snacks, } \\
\text { fats and alcohol }\end{array}$ & $\begin{array}{l}\text { Sweets and } \\
\text { desserts }\end{array}$ & $\begin{array}{l}\text { Refined } \\
\text { grains }\end{array}$ & $\begin{array}{l}\text { Breakfast } \\
\text { cereal }\end{array}$ & $\begin{array}{l}\text { High-fat dairy } \\
\text { products }\end{array}$ \\
\hline$n$ (\% women in cluster) & $204(66.7)$ & $246\left(51.3^{\mathrm{a}}\right)$ & $134\left(52.1^{\mathrm{a}}\right)$ & $125\left(50.6^{\mathrm{a}}\right)$ & $128\left(46.9^{\mathrm{a}}\right)$ & $141\left(57.3^{\mathrm{a}}\right)$ \\
\hline \multicolumn{7}{|l|}{ Characteristics } \\
\hline Age (years) ${ }^{\mathrm{b}}$ & $75.0 \pm 0.2$ & $74.7 \pm 0.2$ & $75.0 \pm 0.3$ & $74.9 \pm 0.2$ & $75.4 \pm 0.2$ & $75.4 \pm 0.2$ \\
\hline Race (\% white) & 77.9 & $44.3^{\mathrm{a}}$ & 83.6 & $52.0^{\mathrm{a}}$ & 68.8 & $63.1^{\mathrm{a}}$ \\
\hline Education $(\% \text { completed high school })^{\mathrm{c}}$ & 91.7 & $74.8^{\mathrm{a}}$ & 90.3 & $66.4^{\mathrm{a}}$ & $81.3^{\mathrm{a}}$ & $83.7^{\mathrm{a}}$ \\
\hline Smoking (lifetime pack-years) ${ }^{\mathrm{c}}$ & $9.5 \pm 1.3$ & $16.5 \pm 1.8^{\mathrm{a}}$ & $13.4 \pm 1.9$ & $11.4 \pm 1.9$ & $9.1 \pm 1.6$ & $9.8 \pm 1.7$ \\
\hline Alcohol (\% any consumption $)^{\mathrm{c}}$ & 55.9 & $44.3^{\mathrm{a}}$ & 60.5 & $36.8^{\mathrm{a}}$ & $42.2^{\mathrm{a}}$ & $44.0^{\mathrm{a}}$ \\
\hline Physical activity (kcal/week) ${ }^{\mathrm{c}}$ & $989 \pm 107$ & $659 \pm 63^{a}$ & $765 \pm 85$ & $638 \pm 97$ & $811 \pm 141$ & $859 \pm 149$ \\
\hline \multicolumn{7}{|l|}{ PPAR $-\gamma$ Pro12Ala genotype $[n(\%)]^{\mathrm{d}}$} \\
\hline Pro/Pro & $166(83.0)$ & $219\left(91.3^{\mathrm{a}}\right)$ & $106(80.3)$ & $109(89.3)$ & $107(84.9)$ & $113(81.9)$ \\
\hline Ala/Pro and Ala/Ala & $34(17.0)$ & $21\left(8.8^{\mathrm{a}}\right)$ & $26(19.7)$ & $13(10.7)$ & $19(15.1)$ & $25(18.1)$ \\
\hline \multicolumn{7}{|l|}{ Dietary factors ${ }^{\mathrm{b}}$} \\
\hline Total calorie intake (kcal) & $1,566 \pm 33$ & $1,707 \pm 39^{\mathrm{a}}$ & $1,873 \pm 46^{\mathrm{a}}$ & $1,695 \pm 61$ & $1,542 \pm 47$ & $1,703 \pm 45$ \\
\hline$\%$ kcal from carbohydrate & $57.6 \pm 0.5$ & $49.8 \pm 0.5^{\mathrm{a}}$ & $52.2 \pm 0.5^{\mathrm{a}}$ & $53.2 \pm 0.6^{\mathrm{a}}$ & $60.4 \pm 0.6^{\mathrm{a}}$ & $51.5 \pm 0.6^{\mathrm{a}}$ \\
\hline$\%$ kcal from protein & $16.7 \pm 0.3$ & $13.9 \pm 0.2^{\mathrm{a}}$ & $12.9 \pm 0.2^{\mathrm{a}}$ & $13.5 \pm 0.2^{\mathrm{a}}$ & $14.0 \pm 0.2^{\mathrm{a}}$ & $14.8 \pm 0.2^{\mathrm{a}}$ \\
\hline$\%$ kcal from fat & $27.4 \pm 0.4$ & $37.3 \pm 0.5^{\mathrm{a}}$ & $36.3 \pm 0.5^{\mathrm{a}}$ & $34.5 \pm 0.6^{\mathrm{a}}$ & $27.9 \pm 0.6$ & $35.2 \pm 0.5^{\mathrm{a}}$ \\
\hline$\%$ kcal from saturated fat & $7.5 \pm 0.1$ & $10.0 \pm 0.1^{\mathrm{a}}$ & $10.7 \pm 0.2^{\mathrm{a}}$ & $9.4 \pm 0.2^{\mathrm{a}}$ & $7.9 \pm 0.2$ & $11.4 \pm 0.2^{\mathrm{a}}$ \\
\hline Total dietary fiber $(\mathrm{g})$ & $19.3 \pm 0.5$ & $15.8 \pm 0.4^{\mathrm{a}}$ & $15.9 \pm 0.5^{\mathrm{a}}$ & $15.5 \pm 0.6^{\mathrm{a}}$ & $16.7 \pm 0.6^{\mathrm{a}}$ & $15.7 \pm 0.6^{\mathrm{a}}$ \\
\hline Dietary glycemic index (glucose scale) & $53.8 \pm 0.2$ & $54.9 \pm 0.3^{\mathrm{a}}$ & $55.2 \pm 0.3^{\mathrm{a}}$ & $57.9 \pm 0.3^{\mathrm{a}}$ & $59.4 \pm 0.3^{\mathrm{a}}$ & $55.4 \pm 0.3^{\mathrm{a}}$ \\
\hline Dietary glycemic load (glucose scale) & $111.1 \pm 2.7$ & $108.4 \pm 2.9$ & $126.3 \pm 3.4^{\mathrm{a}}$ & $121.7 \pm 4.8$ & $127.9 \pm 4.1^{\mathrm{a}}$ & $112.7 \pm 3.3$ \\
\hline Healthy eating index score & $80.8 \pm 0.5$ & $65.9 \pm 0.7^{\mathrm{a}}$ & $64.8 \pm 1.0^{\mathrm{a}}$ & $67.3 \pm 1.0^{\mathrm{a}}$ & $73.3 \pm 0.8^{\mathrm{a}}$ & $69.9 \pm 1.0^{\mathrm{a}}$ \\
\hline
\end{tabular}

Mean \pm SEM, unless otherwise specified

a Significantly different from the 'Healthy foods' cluster, $P \leq 0.05$ (Dunnett's test for continuous variables and chi-square test for categorical variables)

${ }^{b}$ Values from year 2 of the Health ABC study

c Values from baseline of the Health $\mathrm{ABC}$ study

${ }^{\mathrm{d}}$ Genotype information not available for 20 women

Table 4 Multivariate-adjusted means of selected body composition measures in men by dietary pattern cluster

\begin{tabular}{lcccrrr}
\hline & $\begin{array}{l}\text { Healthy foods } \\
\text { (reference) }\end{array}$ & $\begin{array}{l}\text { Meat, snacks, fats } \\
\text { and alcohol }\end{array}$ & \multicolumn{1}{c}{$\begin{array}{l}\text { Sweets and } \\
\text { desserts }\end{array}$} & \multicolumn{1}{c}{$\begin{array}{l}\text { Refined } \\
\text { grains }\end{array}$} & $\begin{array}{l}\text { Breakfast } \\
\text { cereal }\end{array}$ & \multicolumn{1}{c}{$\begin{array}{l}\text { High-fat dairy } \\
\text { products }\end{array}$} \\
\hline$n$ & 102 & 234 & 123 & 122 & 145 & 105 \\
BMI $\left(\mathrm{kg} / \mathrm{m}^{2}\right)$ & $26.1 \pm 0.4$ & $26.7 \pm 0.2$ & $26.4 \pm 0.3$ & $26.4 \pm 0.4$ & $27.1 \pm 0.3$ & $26.6 \pm 0.4$ \\
Total body fat $(\%)$ & $27.9 \pm 0.5$ & $29.4 \pm 0.3^{\mathrm{a}}$ & $29.2 \pm 0.5$ & $28.8 \pm 0.5$ & $30.1 \pm 0.4^{\mathrm{a}}$ & $29.1 \pm 0.5$ \\
Abdominal circumference $(\mathrm{cm})$ & $97.8 \pm 1.1$ & $100.4 \pm 0.7$ & $99.9 \pm 1.0$ & $100.2 \pm 1.0$ & $100.8 \pm 0.9$ & $99.8 \pm 1.1$ \\
Sagittal diameter $(\mathrm{cm})$ & $21.6 \pm 0.3$ & $22.4 \pm 0.2$ & $22.0 \pm 0.3$ & $22.0 \pm 0.3$ & $22.5 \pm 0.2$ & $22.5 \pm 0.3$ \\
Abdominal visceral fat $\left(\mathrm{cm}^{2}\right)$ & $135.4 \pm 6.5$ & $154.3 \pm 4.2$ & $148.1 \pm 5.8$ & $144.1 \pm 5.9$ & $157.0 \pm 5.4^{\mathrm{a}}$ & $148.7 \pm 6.2$ \\
Right thigh intermuscular fat $\left(\mathrm{cm}^{2}\right)$ & $8.7 \pm 0.6$ & $9.4 \pm 0.4$ & $8.9 \pm 0.5$ & $9.2 \pm 0.5$ & $9.8 \pm 0.5$ & $9.5 \pm 0.5$ \\
\hline
\end{tabular}

Least squares mean \pm SEM. Adjusted for age, race, clinical site, education, physical activity, smoking status and total calorie intake

a Significantly different from the 'Healthy foods' cluster, $P \leq 0.05$ (Dunnett's test)

not differ significantly in body composition from those in other clusters, men with the Ala allele in the 'Healthy foods' cluster had significantly lower levels of all measures of adiposity than those in other clusters. Women with the Ala allele in the 'Healthy foods' cluster differed only in right thigh intermuscular fat from those in one other cluster.

Previous studies have found interactions between diet and PPAR- $\gamma$ Pro12Ala genotype in relation to body composition, but results have been inconsistent. Some studies, 
Table 5 Multivariate-adjusted means of selected body composition measures in men by dietary pattern cluster and PPAR- $\gamma$ Pro12Ala genotype

\begin{tabular}{|c|c|c|c|c|c|c|}
\hline & $\begin{array}{l}\text { Healthy foods } \\
\text { (reference) }\end{array}$ & $\begin{array}{l}\text { Meat, snacks, } \\
\text { fats and alcohol }\end{array}$ & $\begin{array}{l}\text { Sweets and } \\
\text { desserts }\end{array}$ & $\begin{array}{l}\text { Refined } \\
\text { grains }\end{array}$ & $\begin{array}{l}\text { Breakfast } \\
\text { cereal }\end{array}$ & $\begin{array}{l}\text { High-fat dairy } \\
\text { products }\end{array}$ \\
\hline Pro/Pro $(n)$ & 79 & 203 & 95 & 106 & 110 & 72 \\
\hline BMI $\left(\mathrm{kg} / \mathrm{m}^{2}\right)$ & $26.2 \pm 0.4$ & $26.4 \pm 0.3$ & $26.4 \pm 0.4$ & $26.2 \pm 0.4$ & $26.7 \pm 0.4$ & $26.4 \pm 0.4$ \\
\hline Total body fat (\%) & $28.3 \pm 0.6$ & $29.1 \pm 0.4$ & $29.1 \pm 0.5$ & $28.4 \pm 0.5$ & $29.5 \pm 0.5$ & $29.0 \pm 0.6$ \\
\hline Abdominal circumference $(\mathrm{cm})$ & $98.3 \pm 1.3$ & $99.6 \pm 0.8$ & $99.9 \pm 1.1$ & $98.7 \pm 1.1$ & $99.1 \pm 1.1$ & $99.0 \pm 1.3$ \\
\hline Sagittal diameter $(\mathrm{cm})$ & $21.9 \pm 0.3$ & $22.2 \pm 0.2$ & $22.0 \pm 0.3$ & $21.9 \pm 0.3$ & $22.2 \pm 0.3$ & $22.4 \pm 0.3$ \\
\hline Abdominal visceral fat $\left(\mathrm{cm}^{2}\right)$ & $138.7 \pm 7.4$ & $148.3 \pm 4.6$ & $148.8 \pm 6.7$ & $142.4 \pm 6.5$ & $150.1 \pm 6.3$ & $143.3 \pm 7.6$ \\
\hline Right thigh intermuscular fat $\left(\mathrm{cm}^{2}\right)$ & $9.2 \pm 0.6$ & $9.1 \pm 0.4$ & $8.6 \pm 0.6$ & $9.3 \pm 0.5$ & $9.2 \pm 0.5$ & $9.4 \pm 0.6$ \\
\hline Ala/Pro and Ala/Ala $(n)$ & 21 & 29 & 21 & 14 & 33 & 29 \\
\hline BMI $\left(\mathrm{kg} / \mathrm{m}^{2}\right)$ & $24.9 \pm 0.8$ & $28.3 \pm 0.7^{\mathrm{a}}$ & $26.3 \pm 0.8$ & $27.6 \pm 1.0$ & $28.2 \pm 0.6^{\mathrm{a}}$ & $27.2 \pm 0.7$ \\
\hline Total body fat (\%) & $25.8 \pm 1.2$ & $31.6 \pm 0.9^{\mathrm{a}}$ & $29.2 \pm 1.1$ & $29.9 \pm 1.3$ & $31.9 \pm 0.9^{\mathrm{a}}$ & $30.1 \pm 1.0^{\mathrm{a}}$ \\
\hline Abdominal circumference $(\mathrm{cm})$ & $95.2 \pm 3.0$ & $104.0 \pm 2.5$ & $100.6 \pm 2.9$ & $109.9 \pm 3.5^{\mathrm{a}}$ & $103.6 \pm 2.3$ & $103.2 \pm 2.5$ \\
\hline Sagittal diameter $(\mathrm{cm})$ & $20.4 \pm 0.6$ & $23.9 \pm 0.5^{\mathrm{a}}$ & $22.1 \pm 0.6$ & $22.5 \pm 0.7$ & $23.4 \pm 0.5^{\mathrm{a}}$ & $23.0 \pm 0.5^{\mathrm{a}}$ \\
\hline Abdominal visceral fat $\left(\mathrm{cm}^{2}\right)$ & $123.2 \pm 14.1$ & $192.8 \pm 11.7^{\mathrm{a}}$ & $152.8 \pm 13.6$ & $154.2 \pm 16.8$ & $185.9 \pm 10.9^{\mathrm{a}}$ & $164.4 \pm 11.9$ \\
\hline Right thigh intermuscular fat $\left(\mathrm{cm}^{2}\right)$ & $7.4 \pm 1.4$ & $11.2 \pm 1.2^{\mathrm{a}}$ & $10.4 \pm 1.3$ & $8.3 \pm 1.7$ & $11.7 \pm 1.1$ & $9.4 \pm 1.2$ \\
\hline
\end{tabular}

Least squares mean \pm SEM. Adjusted for age, race, clinical site, education, physical activity, smoking status and total calorie intake

a Significantly different from the 'Healthy foods' cluster, $P \leq 0.05$ (Dunnett's test)

Table 6 Multivariate-adjusted means of selected body composition measures in women by dietary pattern cluster and PPAR- $\gamma$ Pro12Ala genotype

\begin{tabular}{lccccc}
\hline & $\begin{array}{l}\text { Healthy foods } \\
\text { (reference) }\end{array}$ & $\begin{array}{l}\text { Meat, snacks, } \\
\text { fats and alcohol }\end{array}$ & $\begin{array}{l}\text { Sweets and } \\
\text { desserts }\end{array}$ & $\begin{array}{l}\text { Refined } \\
\text { grains }\end{array}$ & $\begin{array}{l}\text { Breakfast } \\
\text { cereal }\end{array}$ \\
\hline Pro/Pro $(n)$ & 166 & 219 & 106 & 109 & 107 \\
BMI $\left(\mathrm{kg} / \mathrm{m}^{2}\right)$ & $27.5 \pm 0.4$ & $27.7 \pm 0.3$ & $26.5 \pm 0.5$ & $26.9 \pm 0.5$ & $26.8 \pm 0.5$ \\
products
\end{tabular}

Least squares mean \pm SEM. Adjusted for age, race, clinical site, education, physical activity, smoking status and total calorie intake

a Significantly different from the 'Healthy foods' cluster, $P \leq 0.05$ (Dunnett's test)

including the current one, suggest that Ala allele-carriers may be more sensitive to the composition of the diet than Pro/Pro homozygotes, while other studies indicate the reverse. In the Nurses' Health Study, Pro/Pro homozygous women in the highest quintile of total fat intake had a significantly higher BMI than those in the lowest quintile, while Ala allele-carriers showed no relationship between total fat intake and BMI [28]. However, monounsaturated fat intake was not associated with BMI among Pro/Pro homozygotes, but was inversely associated with BMI among Ala allele-carriers. In the Québec Family Study, which included men and women, total fat and saturated fat 
intake were positively associated with waist circumference in Pro/Pro homozygotes but not in Ala allele-carriers [29]. Also, in a study by Adamo et al. [30] of obese women on a 900-kcal formula diet, the Ala variant was associated with resistance to diet-induced weight loss.

In addition to the current study, several others have implied that diet may affect the body composition of Ala allele-carriers more than that of Pro/Pro homozygotes. In the Isle of Ely Study, which included men and women, the dietary polyunsaturated fat to saturated fat ratio was inversely related to BMI among Ala allele-carriers but not Pro/Pro homozygotes [31]. There was no interaction between total fat intake and PPAR- $\gamma$ Pro12Ala genotype in relation to BMI, however. Furthermore, in a diet and exercise intervention in men and women with impaired glucose tolerance, Ala/Ala homozygotes lost more weight than Pro allele-carriers [32]. Similarly, Ala allele-carriers in the weight-loss lifestyle intervention group of the Diabetes Prevention Program lost more weight than Pro/ Pro homozygotes [33]. In another study of men and women with type 2 diabetes, BMI was similar in Ala carriers and Pro/Pro homozygotes in the lower quartile of energy intake but significantly higher in Ala carriers in the upper quartile [34]. Ala allele-carriers were found to have a significantly lower energy intake per kilogram body weight than Pro/Pro homozygotes, and it was suggested that Ala allele-carriers might have a higher food efficiency. In a study of Hispanic American men and women, the Ala allele was associated with increased BMI in those with high intake of polyunsaturated fat, or a high polyunsaturated fat to saturated fat ratio, in an initial model, but not in a subsequent model [18]. Additionally, in a study of overweight women on a hypocaloric diet, weight loss was similar in Ala allele-carriers and Pro/Pro homozygotes, but weight regain during follow-up was greater in Ala allele-carriers [35].

Results of studies have thus been inconsistent and indicate that other factors are likely influencing the relationships among diet, PPAR- $\gamma$ Pro12Ala genotype, and body composition. While gender and weight status may play a role, their impact is not clear from studies to date. The mechanisms behind the effects of the PPAR- $\gamma$ Pro12Ala genotype are also uncertain. Research at the cellular level has associated the Ala variant with reduced PPAR $-\gamma$ transcriptional activity compared to the Pro variant $[36,37]$. Surprisingly, both activation of PPAR- $\gamma$ by thiazolidinediones and reduced transcriptional activity of PPAR- $\gamma$ due to the Pro12Ala polymorphism have been linked to greater insulin sensitivity $[15,16,38-43]$. It is thought that different metabolic pathways mediate the insulin sensitizing effects of both increased and moderately decreased PPAR- $\gamma$ activity. In the current study, men with the Ala allele may have shown stronger associations between diet and body composition due to potentially higher insulin sensitivity, although this could not be inferred as insulin sensitivity was not examined in this study.

A recent study which investigated the mechanistic effects of the PPAR- $\gamma$ Pro12Ala genotype in a mouse model may shed some light on results of the current study [44]. Compared to Pro/Pro homozygotes, Ala/Ala homozygous mice on a standard chow diet had lower body fat mass as well as other metabolic advantages. When the mice were given a high-fat diet, however, the protective effect of the Ala allele was lost, and Ala/Ala homozygous mice had slightly higher body fat mass than Pro/Pro homozygotes. These results were partially attributed to diet-dependent effects of the PPAR- $\gamma$ Pro12Ala genotype on adiponectin signaling and on the interaction of PPAR- $\gamma 2$ with several transcriptional coregulators.

Another potential reason for discrepancies in results of studies to date on diet and PPAR- $\gamma$ Pro12Ala genotype in relation to body composition is that polymorphisms in the PPAR- $\gamma$ gene other than the Pro12Ala polymorphism could influence body composition and be in linkage disequilibrium with the Pro12Ala polymorphism. Linkage disequilibrium patterns can differ among geographically and ethnically distinct populations, and this could contribute to inconsistent results when polymorphisms are considered in isolation $[45,46]$.

Strengths of this study include its unique age group and thorough measures of body composition. While several studies have examined associations between dietary patterns and anthropometric measures of adiposity, this study is unique in assessing dietary patterns of older adults in relation to more detailed measures of adiposity, by CT scan and DEXA, in addition to anthropometric assessments. A possible limitation of this study is that the sample size did not allow subdivision of the study population beyond gender and PPAR- $\gamma$ Pro12Ala genotype in the analyses. Also, as in many other studies of dietary patterns, dietary intake in the current study was measured by FFQ. This method involves several assumptions, including recipes used for certain foods that may not entirely reflect actual intake [47].

In conclusion, the current and previous studies suggest that at least in certain populations, the relationship between diet and body composition differs according to PPAR- $\gamma$ Pro12Ala genotype. Additional genetic and lifestyle factors which influence the relationships of diet, PPAR- $\gamma$ Pro12Ala genotype, and body composition still need to be identified, as do the underlying mechanisms and the specific populations affected. If these questions can be elucidated, eventually diets could be tailored to persons with specific genotypes to minimize their risks of adverse health conditions and promote optimal health. 
Acknowledgments ALA, NRS and TBH were responsible for the study concept and research design. ALA and NRS drafted the article. DKH, FAT, JSL, DES and TBH critically reviewed the article and contributed important intellectual content. National Institute on Aging contracts N01-AG-6-2101, N01-AG-6-2103, and N01-AG-6-2106. This research was supported in part by the Intramural Research Program of the NIH, National Institute on Aging.

Conflict of interest statement None of the authors had a conflict of interest.

Open Access This article is distributed under the terms of the Creative Commons Attribution Noncommercial License which permits any noncommercial use, distribution, and reproduction in any medium, provided the original author(s) and source are credited.

\section{References}

1. Adams KF, Schatzkin A, Harris TB, Kipnis V, Mouw T, BallardBarbash R, Hollenbeck A, Leitzmann MF (2006) Overweight, obesity, and mortality in a large prospective cohort of persons 50 to 71 years old. N Engl J Med 355(8):763-778

2. Moore SC, Mayne ST, Graubard BI, Schatzkin A, Albanes D, Schairer C, Hoover RN, Leitzmann MF (2008) Past body mass index and risk of mortality among women. Int $\mathrm{J}$ Obes (Lond) 32(5):730-739

3. Wannamethee SG, Shaper AG, Lennon L, Whincup PH (2007) Decreased muscle mass and increased central adiposity are independently related to mortality in older men. Am J Clin Nutr 86(5):1339-1346

4. Zhang X, Shu XO, Yang G, Li H, Cai H, Gao YT, Zheng W (2007) Abdominal adiposity and mortality in Chinese women. Arch Intern Med 167(9):886-892

5. Lindqvist $\mathrm{P}$, Andersson K, Sundh V, Lissner L, Björkelund C, Bengtsson C (2006) Concurrent and separate effects of body mass index and waist-to-hip ratio on 24-year mortality in the Population Study of Women in Gothenburg: evidence of age-dependency. Eur J Epidemiol 21(11):789-794

6. Turcato E, Bosello O, Di Francesco V, Harris TB, Zoico E, Bissoli L, Fracassi E, Zamboni M (2000) Waist circumference and abdominal sagittal diameter as surrogates of body fat distribution in the elderly: their relation with cardiovascular risk factors. Int J Obes Relat Metab Disord 24(8):1005-1010

7. Menke A, Muntner P, Wildman RP, Reynolds K, He J (2007) Measures of adiposity and cardiovascular disease risk factors. Obesity (Silver Spring) 15(3):785-795

8. Tuomilehto J, Lindström J, Eriksson JG, Valle TT, Hämäläinen $\mathrm{H}$, Ilanne-Parikka $\mathrm{P}$, Keinänen-Kiukaanniemi S, Laakso $\mathrm{M}$, Louheranta A, Rastas M, Salminen V, Uusitupa M, Finnish Diabetes Prevention Study Group (2001) Prevention of type 2 diabetes mellitus by changes in lifestyle among subjects with impaired glucose tolerance. N Engl J Med 344(18):13431350

9. O'Rahilly S, Barroso I, Wareham NJ (2005) Genetic factors in type 2 diabetes: the end of the beginning? Science 307(5708): 370-373

10. Canizales-Quinteros S, Aguilar-Salinas CA, Ortiz-López MG, Rodríguez-Cruz M, Villarreal-Molina MT, Coral-Vázquez R, Huertas-Vázquez A, Hernández-Caballero A, López-Alarcón M, Brito-Zurita OR, Domínguez-Banda A, Martinez-Sánchez LR, Canto-de Cetina T, Vilchis-Dorantes G, Rosas-Vargas H, Granados-Silvestre MA, Medeiros-Domingo A, Menjivar M, Tusié-Luna MT (2007) Association of PPARG2 Pro12Ala variant with larger body mass index in Mestizo and Amerindian populations of Mexico. Hum Biol 79(1):111-119

11. Kim K, Lee S, Valentine RJ (2007) Association of pro12Ala polymorphism in the peroxisome proliferative-activated receptor gamma2 gene with obesity and hypertension in Korean women. J Nutr Sci Vitaminol (Tokyo) 53(3):239-246

12. Danawati CW, Nagata M, Moriyama H, Hara $K$, Yasuda $H$, Nakayama M, Kotani R, Yamada K, Sakata M, Kurohara M, Wiyono P, Asdie H, Sakaue M, Taniguchi H, Yokono K (2005) A possible association of Pro12Ala polymorphism in peroxisome proliferator-activated receptor gamma2 gene with obesity in native Javanese in Indonesia. Diabetes Metab Res Rev 21(5):465-469

13. Pihlajamaki J, Vanhala M, Vanhala P, Laakso M (2004) The Pro12Ala polymorphism of the PPAR gamma 2 gene regulates weight from birth to adulthood. Obes Res 12(2):187-190

14. Kim KS, Choi SM, Shin SU, Yang HS, Yoon Y (2004) Effects of peroxisome proliferator-activated receptor-gamma 2 Pro12Ala polymorphism on body fat distribution in female Korean subjects. Metabolism 53(12):1538-1543

15. Ghoussaini M, Meyre D, Lobbens S, Charpentier G, Clément K, Charles MA, Tauber M, Weill J, Froguel P (2005) Implication of the Pro12Ala polymorphism of the PPAR-gamma 2 gene in type 2 diabetes and obesity in the French population. BMC Med Genet $6: 11$

16. Gonzalez Sanchez JL, Serrano Rios M, Fernandez Perez C, Laakso M, Martinez Larrad MT (2002) Effect of the Pro12Ala polymorphism of the peroxisome proliferator-activated receptor gamma-2 gene on adiposity, insulin sensitivity and lipid profile in the Spanish population. Eur J Endocrinol 147(4):495-501

17. Kawasaki I, Tahara H, Emoto M, Shoji T, Shioji A, Okuno Y, Inaba M, Nishizawa Y (2002) Impact of Prol2Ala variant in the peroxisome proliferator-activated receptor (PPAR) gamma2 on obesity and insulin resistance in Japanese Type 2 diabetic and healthy subjects. Osaka City Med J 48(1):23-28

18. Nelson TL, Fingerlin TE, Moss L, Barmada MM, Ferrell RE, Norris JM (2007) The PPARgamma Pro12Ala polymorphism is not associated with body mass index or waist circumference among Hispanics from Colorado. Ann Nutr Metab 51(3):252-257

19. Sahyoun NR, Anderson AL, Kanaya AM, Koh-Banerjee P, Kritchevsky SB, de Rekeneire N, Tylavsky FA, Schwartz AV, Lee JS, Harris TB (2005) Dietary glycemic index and load, measures of glucose metabolism, and body fat distribution in older adults. Am J Clin Nutr 82(3):547-552

20. Kennedy ET, Ohls J, Carlson S, Fleming K (1995) The healthy eating index: design and applications. J Am Diet Assoc 95(10):1103-1108

21. Newby PK, Muller D, Hallfrisch J, Qiao N, Andres R, Tucker KL (2003) Dietary patterns and changes in body mass index and waist circumference in adults. Am J Clin Nutr 77(6):14171425

22. Tucker KL, Chen H, Hannan MT, Cupples LA, Wilson PW, Felson D, Kiel DP (2002) Bone mineral density and dietary patterns in older adults: the Framingham Osteoporosis Study. Am J Clin Nutr 76(1):245-252

23. Hill JO, Sidney S, Lewis CE, Tolan K, Scherzinger AL, Stamm ER (1999) Racial differences in amounts of visceral adipose tissue in young adults: the CARDIA (coronary artery risk development in young adults) study. Am J Clin Nutr 69(3):381387

24. Taylor HL, Jacobs DR Jr, Schucker B, Knudsen J, Leon AS, Debacker G (1978) A questionnaire for the assessment of leisure time physical activities. J Chronic Dis 31(12):741-755

25. Health, Aging and Body Composition Study. Genotypes and methodological information. https://psg-mac43.ucsf.edu/ucsf_cc/ version3.1/habc/genotypemethodinfo.asp. Accessed 26 Oct 2009 
26. Ledikwe JH, Smiciklas-Wright H, Mitchell DC, Miller CK, Jensen GL (2004) Dietary patterns of rural older adults are associated with weight and nutritional status. J Am Geriatr Soc 52(4):589-595

27. Newby PK, Muller D, Hallfrisch J, Andres R, Tucker KL (2004) Food patterns measured by factor analysis and anthropometric changes in adults. Am J Clin Nutr 80(2):504-513

28. Memisoglu A, Hu FB, Hankinson SE, Manson JE, De Vivo I, Willett WC, Hunter DJ (2003) Interaction between a peroxisome proliferator-activated receptor gamma gene polymorphism and dietary fat intake in relation to body mass. Hum Mol Genet 12(22):2923-2929

29. Robitaille J, Despres JP, Perusse L, Vohl MC (2003) The PPARgamma P12A polymorphism modulates the relationship between dietary fat intake and components of the metabolic syndrome: results from the Quebec Family Study. Clin Genet 63(2):109-116

30. Adamo KB, Dent R, Langefeld CD, Cox M, Williams K, Carrick KM, Stuart JS, Sundseth SS, Harper ME, McPherson R, Tesson F (2007) Peroxisome proliferator-activated receptor gamma 2 and acyl-CoA synthetase 5 polymorphisms influence diet response. Obesity (Silver Spring) 15(5):1068-1075

31. Luan J, Browne PO, Harding AH, Halsall DJ, O'Rahilly S, Chatterjee VK, Wareham NJ (2001) Evidence for gene-nutrient interaction at the PPARgamma locus. Diabetes 50(3):686-689

32. Lindi VI, Uusitupa MI, Lindström J, Louheranta A, Eriksson JG, Valle TT, Hämäläinen H, Ilanne-Parikka P, Keinänen-Kiukaanniemi S, Laakso M, Tuomilehto J, Finnish Diabetes Prevention Study (2002) Association of the Pro12Ala polymorphism in the PPAR-gamma2 gene with 3-year incidence of type 2 diabetes and body weight change in the Finnish Diabetes Prevention Study. Diabetes 51(8):2581-2586

33. Franks PW, Jablonski KA, Delahanty L, Hanson RL, Kahn SE, Altshuler D, Knowler WC, Florez JC, Diabetes Prevention Program Research Group (2007) The Pro12Ala variant at the peroxisome proliferator-activated receptor gamma gene and change in obesity-related traits in the Diabetes Prevention Program. Diabetologia 50(12):2451-2460

34. Vaccaro O, Lapice E, Monticelli A, Giacchetti M, Castaldo I, Galasso R, Pinelli M, Donnarumma G, Rivellese AA, Cocozza S, Riccardi G (2007) Pro12Ala polymorphism of the PPARgamma2 locus modulates the relationship between energy intake and body weight in type 2 diabetic patients. Diabetes Care 30(5):11561161

35. Nicklas BJ, van Rossum EF, Berman DM, Ryan AS, Dennis KE, Shuldiner AR (2001) Genetic variation in the peroxisome proliferator-activated receptor-gamma2 gene (Pro12Ala) affects metabolic responses to weight loss and subsequent weight regain. Diabetes 50(9):2172-2176

36. Deeb SS, Fajas L, Nemoto M, Pihlajamäki J, Mykkänen L, Kuusisto J, Laakso M, Fujimoto W, Auwerx J (1998) A Pro12Ala substitution in PPARgamma2 associated with decreased receptor activity, lower body mass index and improved insulin sensitivity. Nat Genet 20(3):284-287
37. Masugi J, Tamori Y, Mori H, Koike T, Kasuga M (2000) Inhibitory effect of a proline-to-alanine substitution at codon 12 of peroxisome proliferator-activated receptor-gamma 2 on thiazolidinedione-induced adipogenesis. Biochem Biophys Res Commun 268(1):178-182

38. Yamauchi T, Kamon J, Waki H, Murakami K, Motojima K, Komeda K, Ide T, Kubota N, Terauchi Y, Tobe K, Miki H, Tsuchida A, Akanuma Y, Nagai R, Kimura S, Kadowaki T (2001) The mechanisms by which both heterozygous peroxisome proliferator-activated receptor gamma (PPARgamma) deficiency and PPARgamma agonist improve insulin resistance. J Biol Chem 276(44):41245-41254

39. Memisoglu A, Hu FB, Hankinson SE, Liu S, Meigs JB, Altshuler DM, Hunter DJ, Manson JE (2003) Prospective study of the association between the proline to alanine codon 12 polymorphism in the PPARgamma gene and type 2 diabetes. Diabetes Care 26(10):2915-2917

40. Altshuler D, Hirschhorn JN, Klannemark M, Lindgren CM, Vohl MC, Nemesh J, Lane CR, Schaffner SF, Bolk S, Brewer C, Tuomi T, Gaudet D, Hudson TJ, Daly M, Groop L, Lander ES (2000) The common PPARgamma Pro12Ala polymorphism is associated with decreased risk of type 2 diabetes. Nat Genet 26(1):76-80

41. Buzzetti R, Petrone A, Ribaudo MC, Alemanno I, Zavarella S, Mein CA, Maiani F, Tiberti C, Baroni MG, Vecci E, Arca M, Leonetti F, Di Mario U (2004) The common PPAR-gamma2 Pro12Ala variant is associated with greater insulin sensitivity. Eur J Hum Genet 12(12):1050-1054

42. Sramkova D, Kunesova M, Hainer V, Hill M, Vcelak J, Bendlova B (2002) Is a Pro12Ala polymorphism of the PPARgamma2 gene related to obesity and type 2 diabetes mellitus in the Czech population? Ann N Y Acad Sci 967:265-273

43. Kao WH, Coresh J, Shuldiner AR, Boerwinkle E, Bray MS, Brancati FL, Atherosclerosis Risk in Communities Study (2003) Pro12Ala of the peroxisome proliferator-activated receptorgamma2 gene is associated with lower serum insulin levels in nonobese African Americans: the Atherosclerosis Risk in Communities Study. Diabetes 52(6):1568-1572

44. Heikkinen S, Argmann C, Feige JN, Koutnikova H, Champy MF, Dali-Youcef N, Schadt EE, Laakso M, Auwerx J (2009) The Pro12Ala PPARgamma2 variant determines metabolism at the gene-environment interface. Cell Metab 9(1):88-98

45. Doney A, Fischer B, Frew D, Cumming A, Flavell DM, World M, Montgomery HE, Boyle D, Morris A, Palmer CN (2002) Haplotype analysis of the PPARgamma Pro12Ala and C1431T variants reveals opposing associations with body weight. BMC Genet 3:21

46. Lonjou C, Zhang W, Collins A, Tapper WJ, Elahi E, Maniatis N, Morton NE (2003) Linkage disequilibrium in human populations. Proc Natl Acad Sci USA 100(10):6069-6074

47. Tucker KL (2007) Assessment of usual dietary intake in population studies of gene-diet interaction. Nutr Metab Cardiovasc Dis 17(2):74-81 